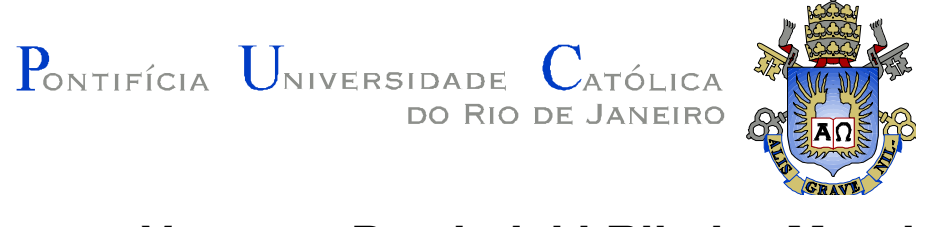

Vanessa Przybylski Ribeiro Magri

Integração de Circuitos de Altas Velocidades por meio de Guia de Onda Semicondutores e Substratos FR-4

Tese de Doutorado

Tese apresentada ao Programa de Pós-Graduação em Engenharia Elétrica da PUC-Rio como requisito parcial para a obtenção do Título de Doutor em Engenharia Elétrica.

Orientador: Marbey Manhães Mosso

Rio de Janeiro

Agosto de 2011 
Vanessa Przybylski Ribeiro Magri

\section{Integração de Circuitos de Altas Velocidades por meio de Guia de Onda Semicondutores e Substratos FR -4}

Tese apresentada como requisito parcial para obtenção do grau de Doutor pelo Programa de Pós-Graduação em Engenharia Elétrica do Departamento de Engenharia Elétrica do Centro Técnico Científico da PUC-Rio. Aprovada pela Comissão Examinadora abaixo assinada.

Prof. Marbey Manhães Mosso

Orientador

Centro de Estudos em Telecomunicações /PUC-Rio

Prof. Gláucio Lima Siqueira

Centro de Estudos em Telecomunicações /PUC-Rio

Profa. Maria Cristina Ribeiro Carvalho

Centro de Estudos em Telecomunicações /PUC-Rio

Prof. Rodolfo Araújo de Azevedo Lima Instituto de Pesquisas da Marinha Prof. Antonio Dias de Macedo Filho Universidade Gama Filho

Prof. Marco Antonio Grivet Mattoso Maia

Centro de Estudos em Telecomunicações /PUC-Rio

Prof. Luiz Alberto de Andrade Instituto de Aeronáutica e Espaço/CTA

Profa. Claudia Barucke Marcondes Paes Leme Centro de Estudos em Telecomunicações /PUC-Rio

Prof. José Eugenio Leal Coordenador Setorial do Centro Técnico Científico - PUC-Rio 
Todos os direitos reservados. É proibida a reprodução total ou parcial do trabalho sem autorização da universidade, da autora e do orientador.

\section{Vanessa Przybylski Ribeiro Magri}

Engenheira Eletricista com ênfase em Telecomunicações, graduada em 2004 na Pontifícia Universidade Católica do Rio de Janeiro. Mestrado concluído em 2007 no CETUC (Centro de Estudos em Telecomunicações PUC-Rio). Doutorado concluído no CETUC em 26 de Agosto de 2011 no laboratório de Sistemas Ópticos e Engenharia de Microondas e Microcircuitos. A atual linha de pesquisa é em Eletromagnetismo avançado, dispositivos de microondas, sistemas de comunicações ópticas e circuitos eletrônicos logicamente programáveis (FPGA).

Ficha Catalográfica

Magri, Vanessa Przybylski Ribeiro
Integração de circuitos de altas
velocidades por meio de guia de onda
semicondutores e substratos FR-4 / Vanessa
Przybylski Ribeiro Magri ; orientador: Marbey
Manhães Mosso. - 2011.
132 f. : il. (color.); 30 cm
Tese (doutorado)-Pontifícia Universidade
Católica do Rio de Janeiro, Departamento de
Engenharia Elétrica, 2011.
Inclui bibliografia

CDD: 621.3 
Dedico o sucesso desta etapa da minha vida a quem sempre me incentivou a enfrentar desafios, ensinou e esteve presente, nos momentos certos e incertos : a DEUS pela graça da vida, principalmente aos meus pais e a minha irmã pela amizade eterna, a toda minha família pelo amor e compreensão, as amigas verdadeiras que me incentivaram também nos momentos em que fui ausente e a alguém que chegou nesta etapa da minha vida com compreensão, respeito, amizade e carinho, meu namorado. 


\section{Agradecimentos}

Ao meu orientador Professor Marbey Manhães Mosso pelos ensinamentos, incentivo e dedicação nesta pesquisa e para minha carreira.

Ao CNPq (Conselho Nacional de Desenvolvimento Científico e Tecnológico).

Ao pesquisador do GSOM/CETUC Rodolfo Lima pela dedicação e ensino durante todos os anos que estudei e trabalhei na PUC-Rio.

Aos pesquisadores e amigos do GSOM/CETUC, Claumir Sarzeda, Henrique Portella, Fernando Diniz, Marcela Silva Novo, Juliana Barros Carvalho, Jorge Angelo Mitrione Souza e Gelza de Moura Barbosa. E também aos amigos do laboratório do PAA/CETUC pelo apoio e amizade.

Ao Pesquisador Juliano Fujioka Mologni, ESSS (software HFSS) .

A Mauricio Kobayashi, Agilent Technologies Brasil (software ADS).

Ao laboratório de Optoeletrônica/CETUC pelo apoio as técnicas de fabricação no CETUC.

A equipe técnica da empresa Lasertools (www.lasertools.com.br/) de São Paulo.

Ao Pesquisador Rudy Massami Sakamoto Kawabata do Laboratório de Semicondutores do CETUC/PUC-Rio (www.labsem.cetuc.puc-rio.br) .

Ao Rogério da Silva Pereira suporte Técnico / Engenharia CETUC .

E aos funcionários do CETUC Marcio José Hote Alves, Marcelo Napoleão Alves e "Chiquinho". 


\section{Resumo}

Magri, Vanessa P. Ribeiro; Mosso, Marbey Manhães. Integração de Circuitos de Altas Velocidades por meio de Guia de Onda Semicondutores e Substratos FR-4. Rio de Janeiro, 2011, 132p. Tese de Doutorado - Departamento de Engenharia Elétrica, Centro de Estudos em Telecomunicações, Pontifícia Universidade Católica do Rio de Janeiro.

Este trabalho de Tese apresenta a pesquisa e desenvolvimento de conexões de ondas guiadas sobre substratos semicondutores ( $\mathrm{SiGe}, \mathrm{GaAs}$ ). A integração de circuitos digitais através de guias S-SIWG (Semiconductor Substrate Integrated Waveguide) utilizando formato de modulação QAM é avaliada e destacada. Conexões internas aos chips e entre chips são associadas com o novo padrão Gigabit Ethernet 802.3ba operando na taxa de $100 \mathrm{Gbit} / \mathrm{s}$ estendendo-se a aplicações de 0,5 - 1,5 Terahertz. É também apresentada a pesquisa e o desenvolvimento de guias e dispositivos de microondas utilizando substratos de baixo custo e altas perdas (FR-4), substratos cerâmicos de alta constante dielétrica $(\mathrm{Er}=80)$ e aplicações em subsistemas híbridos integrados.

\section{Palavras-chave}

Guias de onda planares; Circuitos Integrados; 100 Giga Bit Ethernet; Substratos Semicondutores SiGe, GaAs; FR-4 ; Substratos Cerâmicos 


\section{Abstract}

Magri, Vanessa P. Ribeiro; Mosso, Marbey Manhães (Advisor). High Speed Semiconductor and FR-4 Integrated Waveguide. Rio de Janeiro, 2011, 132 p , Thesis - Departamento de Engenharia Elétrica, Centro de Estudos em Telecomunicações, Pontifícia Universidade Católica do Rio de Janeiro.

This work presents the research, design and development of guided waves connections in semiconductor substrates ( $\mathrm{SiGe}, \mathrm{GaAs})$. The integration of digital systems using Semiconductor Wave Guides (S-SIWG) with QAM modulation formats are highlighted. Ultra-fast inter-chip and inner-chip connections are associated with the new Gigabit Ethernet IEEE 802.3ba standard at 100Gbit/s extended to (0.5-1.5) Terahertz domain. Additionally fiber glass substrates with high losses (Teflon/FR-4) and high dielectric ceramic substrates $(\mathrm{Er}=80)$ are also developed to be integrated with microwave devices, analog printed circuits boards and high Speed digital circuits and systems.

\section{Keywords}

Semiconductor Substrate Integrated Wave Guide, SiGe, GaAs; FR-4 substrate; IEEE 802ba ; High Speed Printed Circuit Board; Integrated Circuits; Electronic Circuits. 


\section{Sumário}

1. Introdução 17

2. Guias de onda em substratos dielétricos 20

2.1. Guias NRD 20

2.2. Modelo guias RWG x SIWG 24

2.3. Estruturas de transição entre linhas planares e guias SIWG 27

2.3.1. Transição Microstrip - SIWG 27

2.3.2. Transição Coaxial - SIWG 28

2.4. Comentários 30

3. Subsistemas de Ondas Guiadas 31

3.1. FR-4 SIWG 31

3.2. FR-4 PCB em 10Gb/s 37

3.3. Filtro SIWG no FR-4 centrado em $10 \mathrm{GHz}$

3.4. Ondas guiadas em substratos de Altíssimas constantes dielétricas 46

3.5. Comentários 50

4. Utilização de guias semicondutores em circuitos integrados no domínio do Gigabyte e Terabyte. 52

4.1. Interfaces eletrônicas Ultra Rápidas 52

4.2. Conexões utilizando configurações S-SIWG 57

4.2.1. Implementação em Substrato de Arseneto de Gálio (GaAs). $\quad 57$

4.2.2. Implementação em Substrato de Silicio Germânio (SiGe) 60

4.2.3. Avaliação do SiGe - SIWG na faixa de freqüência de (40-160) GHz 62

4.2.4. Avaliação das Perdas em configurações SIWG e linhas microstrip 64

4.3. Excitação de conexões SIWG por meio de adaptadores

$\begin{array}{ll}\text { Coaxial - SIWG } & 67\end{array}$

4.4. Conexões de sinais digitais utilizando módulos SIWG na taxa de 100 Gbit/s em substratos semicondutores 72

$\begin{array}{ll}\text { 4.5. Comentários } & 74\end{array}$ 
5. Protótipo de guia de onda operando na faixa de freqüências de $(15-60) \mathrm{GHz} \quad 76$

5.1. R6010 -SIWG 76

5.2. Protótipo SiGe-SIWG de $15 \mathrm{GHz}$ a $60 \mathrm{GHz}$

5.3. Comentários 89

6. Comentários finais e conclusões 91

6.1. Observações Finais 91

6.2. Principais Resultados Obtidos 92

6.3. Trabalhos Futuros 93

7. Referências Bibliográficas 94

$\begin{array}{ll}\text { Anexo } 1 \text { - Condições de Contorno guia NRD } & 97\end{array}$

Equações de propagação no meio dielétrico homogêneo $\begin{array}{ll}\text { sem fontes e sem cargas } & 97\end{array}$

Condições de Contorno em um guia dielétrico formado por 2 meios dielétricos sem fontes e sem cargas de paredes metálicas 98

A) Modos de propagação LSEx (Longitudinal Section Electric) ou TEx (Transversal Electric) em relação a $x$ :

100

B) Modos de propagação LSMx (Longitudinal Section Magnetic)

ou TMx (Transversal Magnetic) em relação a x :

102

Anexo 2 - Teorema de Floquet 105

Anexo 3 - Diferentes configurações das vias do guia NRD 
Anexo 7 - Publicação 3

Anexo 8 : GB Ethernet $\quad 125$

Ethernet $1 \mathrm{~Gb} / \mathrm{s} \quad 126$

Ethernet $10 \mathrm{~Gb} / \mathrm{s} \quad 127$

Ethernet $100 \mathrm{~Gb} / \mathrm{s}$ e $400 \mathrm{~Gb} / \mathrm{s}$

Anexo 9 - Resultado de medidas Protótipo SIWG R6010 131 


\section{Lista de Figuras}

Figura 1 - Condições de Contorno de um guia dielétrico retangular ideal.

Figura 2 - Configuração tridimensional do guia NRD ;

(a) Modelo de guia dielétrico retangular ideal ;

(b) Modelo real do guia NRD.

Figura 3 - Aproximação pelo Teorema de Floquet para a propagação

no guia NRD. 22

Figura 4 - Modelo tridimensional do guia NRD em Alumina simulado no CST. 23

Figura 5 - Simulação Eletromagnética para o guia NRD em Alumina. 24

Figura 6 - Modelo tridimensional dos guias de onda: (a) SIWG 3D ;

(b) modelo RWG. 25

Figura 7 - Transição microstrip para SIWG [6]. 28

Figura 8 - Modelo tridimensional de um guia SIWG excitado por probe coaxial. 28

Figura 9 - Corte longitudinal do guia SIWG no centro do coaxial.

Figura 10 - Modelo do guia em FR4 ; (a) Modelo eletromagnético 3D ;

(b) Modelo RWG.

Figura 11 - Resultados da simulação eletromagnética (a) SIWG 3D;

(b) Modelo equivalente RWG

Figura 12 - Modelo 3D do guia FR-4 SIWG com transição microstrip;

(a) parede com uma fileira de furos; (b) parede com duas fileiras de furos no HFSS [19];

Figura 13 - Resultado de simulação eletromagnética do modelo 3D

do FR-4 SIWG com transição microstrip comparação da parede do guia. 34

Figura 14 - Protótipo SIWG com transição microstrip.

Figura 15 - resultado simulação SWG comparado com

resultado experimental.

Figura 16 - Comportamento de Fase do FR4 - SIWG , comparação

de simulação EM e medição experimental.

Figura 17 -Retardo de grupo do protótipo FR4-SIWG, resultado medido.

Figura 18 - Setup experimental para teste da transmissão digital

PRBS 2.5Gbits/s no guia FR-4 SIWG na portadora de $10 \mathrm{GHz}$

Figura 19 - Espectro digital medido antes e depois do guia FR-4 SIWG 
na configuração da figura 18.

Figura 20 - Diagrama de olho para a transmissão do sinal PRBS

de 2.5 Gbits/s no guia FR-4 SIWG na configuração da figura 18.

Figura 21 - Transmissão digital serial de 10Gbit/s no FR-4 SIWG com modulação QAM

Figura 22 - Circuito de simulação da transmissão do sinal PRBS 10Gbit/s no formato 16-QAM integrado com o modelo 3D EM do guia FR-4 SIWG

Figura 23 - Espectro simulado para a transmissão do sinal digital PRBS 10Gbit/s no formato 16-QAM : em azul o sinal na entrada do guia e em verde o sinal na saída do guia.

Figura 24 - Diagrama de olho para a transmissão do sinal PRBS

10 Gbits/s modulado em 16-QAM transmitido no guia FR-4 SIWG.

Figura 25 - Filtro SIWG 3D no FR-4

Figura 26 - Modelo equivalente do circuito PI de cada via metalizada centralizada no guia [22].

Figura 27 - Circuito equivalente do filtro SIWG utilizado para simulação

e otimização no ADS

Figura 28 - Comparação entre a simulação EM 3D do filtro SIWG e a simulação do circuito equivalente.

Figura 29 - Protótipo do filtro com transição Microstrip e conectorização, equivalente modelo 3D no CST.

Figura 30 - Comparação entre a simulação do modelo 3D do filtro

e a caracterização experimental do protótipo.

Figura 31 - Resultado experimental da fase e do retardo do filtro SIWG.

Figura 32 - Modelo SIWG $\varepsilon r=80$ : (a) modelo tridimensional;

(b) modelo RWG equivalente.

Figura 33 - Resultado de simulação eletromagnética SIWG $\times$ RWG

no substrato Trans-tech S-8600 para aRWG=aSIWG $=3.3518 \mathrm{~mm}$

Figura 34 - Resultado de simulação eletromagnética SIWG $\times$ RWG no substrato Trans-tech S-8600 para aRWG $=3.3518 \mathrm{~mm}$ e aSIWG $=3.7418 \mathrm{~mm}$.

Figura 35 - Radar de acompanhamento em um circuito 
totalmente integrado pela tecnologia SIWG

Figura 36 - Detalhes da arquitetura 100GBE IEEE802.3ba

indicado uma redução de

Figura 37 - Comportamento passa-baixo dos fluxos digitais

NRZ e RZ (PRBS) atenuados pela resposta de freqüência típica

de um guia de onda e seu comportamento passa-alto.

Figura 38 - Novo perfil das camadas MAC e PHY utilizando

conexões seriais SIWG associados ao padrão 802.3ba.

Figura 39 - Nova Tecnologia de conexão Inter-chip por meio de guias de ondas S-SIWG.

Figura 40 - GaAs-SIWG ; (a) Modelo 3D; (b) modelo RWG equivalente.

Figura 41 - Resposta em freqüência para a perda de retorno e a perda de inserção do modelo do guia GaAs - SIWG comparado com o respectivo modelo RWG na faixa de $50 \mathrm{GHz}$ a $160 \mathrm{GHz}$.

Figura 42 - Resposta em freqüência para a perda de retorno e a perda de inserção do modelo do guia GaAs - SIWG comparado com o respectivo modelo RWG na faixa de $0.5 \mathrm{THz}$ a $1.6 \mathrm{THz}$.

Figura 43 - SiGe-SIWG (a) Modelo 3D EM ; (b) modelo RWG equivalente

Figura 44 - Resposta em freqüência para a perda de inserção e perda de retorno do modelo do guia SiGe-SIWG comparado com o respectivo modelo RWG na faixa de $40 \mathrm{GHz}$ a $160 \mathrm{GHz}$.

Figura 45 - Modos excitados no guia SiGe-SIWG sem transição na banda de $50 \mathrm{GHz}$ a $160 \mathrm{GHz}$.

Figura 46 - Distribuição do campo eletromagnético nas portas do guia SiGe-SIWG; (a) TE10 ; (b) TE20 ; (c) TE01

Figura 47 - Resposta em freqüência de simulação e comparação com referências bibliográficas [33] da linha microstrip no "bulk" de silício comparado com o guia SiGe-SIWG na faixa de $(70-160) \mathrm{GHz}$

Figura 48 - Atenuação em dB/mm de uma linha microstrip e de um guia em bulk de silício na faixa de freqüências de (0.5-1.5) $\mathrm{THz}$.

Figura 49 - Resposta em freqüência da simulação Eletromagnética 3D do SiGe-RWG na faixa de frequências de (0.2-1.6) THz Comparada com o modelo RWG.

Figura 50 - Modelo da Transição guia SiGe-SIWG coaxial $50 \Omega$. 
O trecho coaxial de 30um indicado acima está localizado no interior de uma camada de SiGe com esta espessura.

Figura 51 - Modelo 3D EM do guia SiGe-SIWG com a transição coaxial de $50 \Omega 69$ Figura 52 - Modos excitados no guia SiGe-SIWG com transição coaxial de $50 \Omega$ na banda de $50 \mathrm{GHz}$ a $150 \mathrm{GHz}$.

Figura 53 - Distribuição do campo eletromagnético nos probes coaxial de $50 \Omega$ do guia SiGe-SIWG.

Figura 54 - Resposta em freqüência para a perda de inserção e perda de retorno do guia SiGe-SIWG com transição coaxial de $50 \Omega$

Figura 55 - Comportamento de Fase do SiGe - SIWG com transição coaxial de $50 \Omega$

Figura 56 - Comportamento de retardo de grupo do SiGe -SIWG com transição coaxial de $50 \Omega$

Figura 57 - Setup de simulação para teste da transmissão de um sinal de 100Gbits/s modulado via 16QAM através de um guia SiGe-SIWG incluindo os estágios de "up" e "down converter".

Figura 58 - Espectro simulado para a transmissão do sinal digital PRBS 25Gbit/s; em laranja o sinal na entrada do guia e em vermelho o sinal na saída do guia.

Figura 59 - Diagrama de olho para a transmissão do sinal PRBS 25 Gbits/s transmitido no guia SiGe-SIWG.

Figura 60 - Guia R6010 - SIWG ; (a) modelo 3D EM ; (b) modelo RWG ;

Figura 61 - Guia SIWG - R6010 de $15 \mathrm{GHz}$ a $60 \mathrm{GHz}$ com transições microstrip simulado no HFSS.

Figura 62 - Resposta em freqüência da perda de inserção e da perda de retorno do guia SIWG - R6010 de 15 a 60 GHz / SIMULAÇÃO.

Figura 63 - Protótipos do guia SIWG - R6010 realizado na Prototipadora de circuitos impressos cuja metalização das vias foi manual.

(a) vias metalizadas com epoxy; (b) vias metalizadas com epoxy + fio de protoboard.

Figura 64 - Resposta em freqüência da perda de inserção S21 do protótipo do guia R6010-SIWG (a) modelo 3D EM (b) protótipo com as vias metalizadas em epox ; (c) protótipo com as vias metalizadas com epox + fio de protoboard. 
Figura 65 - Resposta em freqüência da perda de retorno $\mathrm{S} 11$ do protótipo do guia R6010-SIWG medido no analisador de redes vetorial; modelo 3D EM ; (1) protótipo com as vias metalizadas em epox ;

(2) protótipo com as vias metalizadas com epox + fio de protoboard.

Figura 66 - Comportamento da fase do protótipo do guia R6010-SIWG medido no analizador de redes vetorial (1) protótipo com as vias metalizadas em epox ; (2) protótipo com as vias metalizadas com epox + fio de protoboard.

Figura 67 - Retardo de grupo do protótipo do guia R6010-SIWG medido no analizador de redes vetorial (1) protótipo com as vias metalizadas em epox ; (2) protótipo com as vias metalizadas com epox + fio de protoboard.

Figura 68 - Bancada de teste da perda de inserção de 20 a $40 \mathrm{GHz}$

Figura 69 - Resposta em freqüência para a perda de inserção

de 20 a $40 \mathrm{GHz}$ dos dois protótipos do guia R6010-SIWG realizados.

Figura 70 - Modelo 3D EM do guia SiGe-SIWG

Figura 71 - Resposta em freqüência do modelo do guia SiGe-SIWG realizado com transição microstrip.

Figura 72 - Resposta em freqüência da fase do guia SiGe-SIWG realizado com transição microstrip.

Figura 73 - Retardo de grupo do guia SiGe-SIWG realizado com transição microstrip.

Figura 74 - Protótipo do guia SiGe-SIWG realizado sem a metalização.

Figura 75 - Protótipo do guia SiGe-SIWG realizado com a transição microstrip e metalização de 0.5 um de ouro; (a) protótipo conectorizado sendo as vias metalizadas com epox ; (b) protótipo sem conectorização e sem metalização das vias.

Figura 76 - Simulação da Perda de inserção do protótipo em SiGe na faixa de freqüências de (15-60) GHz para diversas espessuras de metalização. 


\section{Lista de Tabelas}

Tabela 1 - Dimensões do guia FR4-SIWG. 32

Tabela 2 - Dimensões do guia de alta constante dielétrica $\mathrm{Er}=80$ no

Substrato Transtech S-8600 [3] 47

Tabela 3 - Taxas de transmissão nos formatos de modulação digital QAM. $\quad 56$

Tabela 4 - Dimensões do GaAs-SIWG para aplicações de $50 \mathrm{GHz}$ a

$150 \mathrm{GHz}$ e também $300 \mathrm{GHz}$ a $1.5 \mathrm{THz}$

Tabela 5 - Dimensões do SiGe-SIWG nas bandas de 50 - $150 \mathrm{GHz}$

e de $0.5-1.5 \mathrm{THz} \quad 61$

Tabela 6 - Dimensões do guia SiGe - SIWG com a transição coaxial /SIWG. 68

Tabela 7 - Dimensões do protótipo do guia no substrato Roger 6010.

Tabela 8 - Dimensões do protótipo do guia SiGe -SIWG 85 